\title{
Rezeptionssignale beim Prüfungsgespräch aus sprechwissenschaftlicher Sicht
}

\section{Einführung}

Das Ziel des Beitrags ist es, die Rezeptionssignale bei einem Prüfungsgespräch aus sprechwissenschaftlicher Sicht zu beschreiben. Untersucht werden kurze (einund zweisilbige) Rückmeldungen, die die Einstellung einer deutschen Prüferin zum Gehörten signalisieren. Genauer gesagt handelt es sich um die Realisierungen von ja, hm_hm und $h m$, die je nach phonetischer Form verschiedene kommunikative Funktionen ausüben können. Aus diesem Grund werden in die Analyse die grundlegenden akustisch-auditiven Parameter einbezogen: Grundfrequenz/ Tonhöhe, Intensität/Lautstärke und Dauer/Länge, die prosodische Phänomene markieren. Prosodie wird dabei nach Selting (1995:1) verstanden als

Oberbegriff für diejenigen suprasegmentalen Aspekte der Rede, die sich aus dem Zusammenspiel der akustischen Parameter Grundfrequenz (F0), Intensität und Dauer in silbengroßen oder größeren Domänen ergeben. Hierzu gehören auditive Phänomene wie Intonation, d. h. der Tonhöhenverlauf gesprochener Sprache in der Zeit, Lautstärke, Länge, Pause, sowie die damit zusammenhängenden komplexeren Phänomene Sprechgeschwindigkeit/ Tempo und Rhythmus. ${ }^{1}$

Da prosodische Gestaltungsmittel stark kontextabhängig sind, wird das Rezeptionsverhalten der Prüferin unter einem situativen und idiolektalen Gesichtspunkt betrachtet. Folgende Faktoren werden dabei berücksichtigt: Gesprächsbeteiligte

\footnotetext{
1 Mehr zur Prosodie siehe ebenfalls Günther (1999:15ff.), Pétursson/Neppert (2002:150ff.), Grassegger (2010:63) und Hirschfeld/Stock (2013:38ff.).
} 
und ihre Beziehung zueinander, Ziel, Ort und Thema der Kommunikation sowie Merkmale der Reziprozität. ${ }^{2}$ Es wird der Frage nachgegangen, inwiefern die phonetische Form die Semantik der Rezeptionssignale im Prüfungsgespräch determiniert. Die Grundlage der Analyse ist die von der Autorin des vorliegenden Beitrags durchgeführte auditive Analyse. Der subjektive Höreindruck wird zum Zweck der Veranschaulichung um akustische Information ergänzt, die in $\mathrm{Praat}^{3}$ dargestellt wird. Die sprechsprachlichen Daten (Audiodatei samt zugehörigem Transkript) stammen aus dem GeWiss-Korpus. ${ }^{4}$ Die Audioaufnahme wurde mit Hilfe von EXMARaLDA ${ }^{5}$ nach den Konventionen des Minimaltranskripts von GAT $2^{6}$ transkribiert.

\section{Prüfungsgespräch im deutschen akademischen Kontext}

Anders als im Falle eines natürlichen Alltagsgesprächs haben die Regeln des Verhaltens in einem institutionell geregelten Gespräch einen bei weitem höheren Grad an Verbindlichkeit (vgl. Brinker/Cölfen/Pappert 2014:143). Die Textsorte Prüfungsgespräch ist funktional („Prüfung“) und dialogisch („Gespräch“) definiert (vgl. Techtmeier 1998:517). Sie ist dabei primär sachbezogen (,SachGespräch“) und asymmetrisch (vgl. Bartsch/Pabst-Weinschenk 2011:125). Die Asymmetrie ist auf die sozialen Rollen der Gesprächspartner zurückzuführen. Diese sind gesetzlich vorgeschrieben und beziehen sich auf die institutionellen Rahmenbedingungen, die das Schema der Kommunikation konstituieren. Der Prüfende ist dazu verpflichtet, die wissenschaftliche Kommunikation zu steuern und die Inhalte hinsichtlich der Sachlichkeit zu kontrollieren. Der Geprüfte folgt den Anregungen des Prüfenden und bekommt mehr und weniger konkrete Fragen gestellt, die er beantworten soll. In diesem Sinne dominiert der Prüfer die Prüfungssituation dank der ihm durch die Institution gegebenen Stellung und initiiert

${ }^{2}$ Für die Sprechwissenschaft sind nach Neuber (2013:115) vor allem die folgenden Gesichtspunkte von Gesprächen analyse- und interpretationswürdig: Gesprächsbeteiligte, Themen, Inhalte und die Sachverhalte, auf die sie sich beziehen, Modalität der Gesprächsführung, Anlass, Ziel, Umgebungsvariablen (Ort, Zeit, außersprachliche Einflüsse, Reize und Signale), Verlaufskategorien (Gesprächsorganisation, Sequenzierung, Phasenbildung), soziale Rollen und psychologisch beschreibbare Besonderheiten, Merkmale der Reziprozität, des Verständigungshandelns sowie der Sinnkonstitution, Schlussfolgerungen für Möglichkeiten der Gesprächsoptimierung.

${ }^{3}$ Siehe http://www.fon.hum.uva.nl/praat/.

${ }^{4}$ GeWiss (Gesprochene Wissenschaftssprache kontrastiv) ist ein Projekt zur Erforschung der deutschen, englischen und polnischen gesprochenen Wissenschaftssprache. Es verfolgt das Ziel, eine empirische Grundlage für vergleichende Untersuchungen in diesem Bereich zu schaffen. Die Autorin dieses Beitrages war Mitglied der polnischen Projektgruppe unter der Leitung von Prof. Dr. Lesław Cirko. Zugang zum GeWiss-Korpus unter https://gewiss.uni-leipzig.de/.

5 Siehe https://exmaralda.org/de/.

6 Siehe http://www.gespraechsforschung-ozs.de/heft2009/px-gat2.pdf. Die Liste der verwendeten Transkriptionszeichen befindet sich im Anhang. 
in der Regel das Prüfungsgespräch; der Prüfling dagegen reagiert (vgl. Techtmeier 1998:518). Trotz der Asymmetrie auf der lokalen Ebene des Gesprächs, die u. a. durch initiierende und respondierende Aspekte der Äußerungen sowie durch den Sprecher- und Hörerstatus entsteht ${ }^{7}$, sind die Prüfer weder die Macht noch besitzen sie die Macht, was Meer (1998) in ihrer Untersuchung zu mündlichen Abschlussprüfungen in der Hochschule explizit zum Ausdruck bringt. Im Hinblick auf die Turnwechselorganisation und das Rückmeldeverhalten scheint es die Aufgabe der Prüfenden zu sein, Kandidaten in ihren Ausführungen zu unterstützen (ebd., S. 49). Dazu gehören u. a. positiv bestätigende Hörerrückmeldungen. ${ }^{8}$ Die Autorin stellt fest, dass „Kandidat/inn/en von ihren Prüfer/inne/n in den Mittelpunkt der Aufmerksamkeit gerückt werden oder sich selbst (möglichst freiwillig) anhand ihres wissenschaftlichen Wissens im Mittelpunkt präsentieren" (ebd., S. 78). Nach Sucharowski (2001:1570f.) kann die Prüfung dann gesprächsähnlichen Charakter annehmen, ,wenn der Geprüfte Möglichkeiten erhält, mit dem Gefragten offen umzugehen. Dazu trägt das Frageverhalten des Prüfers bei, das bis hin zu Formen der Diskussion über ein Thema reicht und dann die vorhandene institutionelle Asymmetrie phasenweise aufhebt". Daraus folgt, dass Asymmetrien nicht a priori institutionell vorgegeben sind, sondern dass sie von den Beteiligten im Gespräch aktualisiert werden. Auch die Verteilung von Wissen/Nichtwissen lässt sich nicht einfach auf der Achse Experte/Laie verteilen, vielmehr liegen je spezifische Wissensbestände vor, die sich in verschiedenen Gesprächsphasen unterscheiden (vgl. Janich/Birkner 2015:211). In diesem Sinne definieren Brock/ Meer (2004:203) Asymmetrie als „kommunikative Ungleichheit in Bezug auf ein spezifisches Kriterium oder Phänomen“.

\section{Zum Begriff Rezeptionssignale}

Die Rezeptionssignale werden als Teil der Gliederungssignale zu Höreraktivitäten im Dialog aufgefasst. Der Terminus ist auf die englische Bezeichnung „,back channel“ zurückzuführen, mit denen der Hörer dem Sprecher seine Aufmerksamkeit und seine Zuhörbereitschaft anzeigt. Die synonymen Bezeichnungen sind: Hörerbzw. Rückmeldungssignale, Rückkopplungs- bzw. Rückmeldeverhalten oder Hörerkommentare. ${ }^{9} \mathrm{Zu}$ den Rezeptionssignalen gehören sprachliche Ausdrücke, u. a.

\footnotetext{
7 Auf der globalen Ebene betreffen Asymmetrien längere Gesprächsphasen sowie ganze Gespräche und Interaktionssituationen, siehe dazu Tiittula (2001:1362).

${ }^{8}$ Dies spiegelt sich in der Tokenzahl der Prüflinge im GeWiss-Teilkorpus zu deutschsprachigen Prüfungsgesprächen mit Muttersprachlern im deutschen akademischen Kontext wider. Die gesamte Tokenzahl der Prüflinge beträgt 65 161; die der Prüfer 47 368, siehe https://gewiss.uni-leipzig.de/ fileadmin/documents/Statistische_Angaben_April2014.pdf.

9 Eine Untergruppe der Rezeptionssignale bilden die Rezeptionspartikeln, Rückmeldepartikeln oder Diskurspartikeln.
} 
Rückmeldungspartikeln oder Kontaktsignale, Satzvervollständigungen, Bitten um Klärung, kurze Statements sowie entsprechende nicht-sprachlichen Signale (z. B. Kopfnicken und -schütteln) (vgl. dazu Bußmann 2008:77 und Glück/Rödel 2016:575). In der Beschreibung der Rezeptionssignale in der Fachliteratur fällt auf, dass sie vielfältige phonetische Formen annehmen und verschiedene kommunikative Funktionen erfüllen können. Fasst man die wichtigsten Eigenschaften der Rezeptionssignale zusammen, ergibt sich folgendes Bild: Die herkömmlichen Rezeptionssignale sind kurze (ein- oder zweisilbige), prosodisch kodierte und redebegleitende Kommentare zum aktuell Gehörten. Mancherlei Rezeptionssignale sind stark automatisiert und verfügen über keine lexikalische Bedeutung. Sie können nach syntaktisch abgeschlossenen Konstruktionen auftreten oder parallel zum Gesprächsbeitrag verlaufen, wobei sie diesen im Normalfall nicht stören oder berühren. Dabei beanspruchen sie das Rederecht nicht. Ihre Hauptfunktion besteht darin, die sprachliche Interaktion zu stützen ggf. zu steuern. Auf der einen Seite signalisieren sie das aktive Zuhören und gelten als aufmerksamkeitsbezeugende Hörersignale. Auf der anderen Seite dienen sie zur Verarbeitung des Gesagten auf einer Skala von Konvergenz und Divergenz und gelten als kommentierende Hörersignale (vgl. dazu Rath 1979:37, Schegloff 1982:81, Ehlich 1986:215, Selting 1995:72ff., Selting 2001:107, Stein 2003:398 ff., Linke/Nussbaumer/Portmann 2004:304ff., Gilles 2005:49ff., Graefen/Liedke 2008:256ff., Duden 2009:1232, Schwitalla 2012:87, Imo/Lanwer 2019:101).

\section{Analyse der Rezeptionssignale im Prüfungsgespräch}

Gegenstand der Untersuchung ist eine 20-minutige Master-Modulprüfung im Fach Phonetik. Am Prüfungsgespräch nehmen eine 52-jährige deutsche Seminarleiterin in der Rolle der Prüferin und eine 26-jährige deutsche Studentin aktiv teil. Das Ziel der Kommunikation ist es herauszufinden, wie fundiert das Wissen des Prüflings und wie ausgeprägt seine Fähigkeit zum wissenschaftlichen Argumentieren sind. Analysiert wird das Rezeptionsverhalten der Prüferin. Im Mittelpunkt der Untersuchung stehen die im Prüfungsgespräch am häufigsten auftretenden Rezeptionssignale: $j a, h m \_h m$ und $h m$. In der Aufnahme wurden insgesamt 132 solcher Vorkommnisse festgestellt: 78 von ja, 34 von $\mathrm{hm} \_\mathrm{hm}$ und 20 von $\mathrm{hm}$. Das bedeutet, dass ca. sieben Rezeptionssignale pro Minute vonseiten der Prüferin produziert wurden. ${ }^{10}$ Diese werden aus sprechwissenschaftlicher Sicht betrachtet, d. h. die einzelnen Realisierungen werden durch die prosodischen Merkmale

${ }^{10}$ Cirko (2016) untersucht Gebrauchsregularitäten und -besonderheiten von Signalen mit phatischer Funktion anhand der GeWiss-Korpora deutscher, englischer und polnischer Prüfungsgespräche und bemerkt, dass der Prüfer dann mehr phatische Signale verwendet, wenn der Prüfling ihn positiv überrascht, ihn in seinen originellen Interpretationsduktus einbezieht und zur echten Diskussion herausfordert (ebd., S. 33). 
Grundfrequenz/Tonhöhe, Intensitä/Lautstärke und Dauer/Länge charakterisiert und anschließend hinsichtlich kommunikativ-pragmatischer Aspekte in vier funktionale Gruppen eingeteilt: Zustimmungssignale, Aufmerksamkeitssignale, Ermunterungssignale und Bestätigungssignale.

\subsection{Zustimmungssignale: $j a$ und $h m \_h m$}

Die größte Gruppe der untersuchten Rezeptionssignale (ca. 52\%) bilden die $\mathrm{Zu}$ stimmungssignale, die explizit mit Hilfe von ja oder implizit mit Hilfe von $\mathrm{hm}$ hm vermittelt werden. Die beiden Realisierungen werden durch eine leicht fallende Grundfrequenz, eine tief fallende Intensität und eine relativ kurze Dauer gekennzeichnet, vgl. Graphik $1^{11}$.

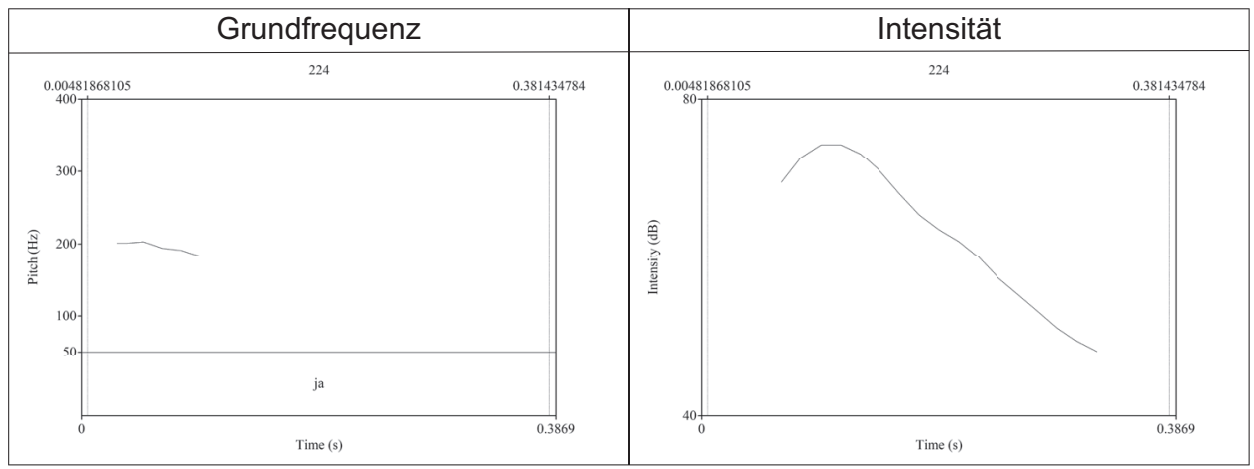

Graphik 1: Grundfrequenz- und Intensitätskonturen von ja in der Funktion eines Zustimmungssignals

Bei ja beobachtet man einen kleinen Abstieg der Grundfrequenz (von ca. 203 bis $183 \mathrm{~Hz}$ ) und einen rasanten Abstieg der Intensität (von ca. 74 bis $48 \mathrm{~dB}$ ). Die Dauer liegt bei ca. 0,2 Sek. Im analysierten Fall schließt das eindeutige $J a$ den Redebeitrag vom Prüfling ab, indem es am Ende einer syntaktischen Einheit (einfacher Satz) und einer prosodischen Einheit (fallende Tonhöhenbewegung ${ }^{12}$ ) erscheint. Die klare Akzeptanz seitens der Prüferin führt dazu, dass die Studentin ihre Aussage fortsetzt, vgl. Dialog 1.

Dialog 1

Studentin: ich kann_s mir grad selbst schwer vorstellen also einfach ähm (4.1) also ich denke da muss ich ne eigene

Prüferin: $h m \_h m$

11 Sämtliche Graphiken wurden in Praat erstellt.

12 Im Transkript zusätzlich durch Semikolon markiert. 
Studentin: repräsentationsebene für (.) aufbauen un die muss halt irgendwie (0.4) bewusst geschehen;

Prüferin: (.) ja (.)

(0.2)

Studentin: un die geschieht (.) wahrscheinlich unter mehreren wegen bewusst

Ähnliche prosodische Parameter werden beim zweisilbigen $H m \_h m$ in derselben Funktion festgestellt: eine leicht fallende Grundfrequenz (von ca. 249 bis $191 \mathrm{~Hz}$ ), immer niedrigere Intensität (von ca. 70 bis $54 \mathrm{~dB}$ ) und eine relativ kurze Dauer (ca. 0,4 Sek), vgl. Graphik 2 und Dialog 2.

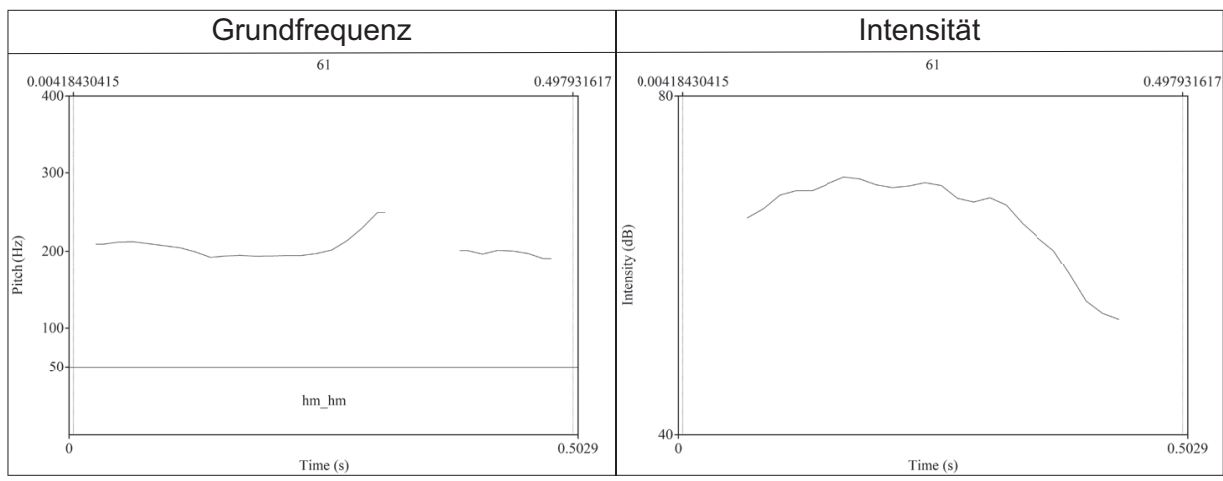

Graphik 2: Grundfrequenz- und Intensitätskonturen von $h m \_h m$ in der Funktion eines Zustimmungssignals

Dialog 2

Prüferin: übrigens ja auch äh $n$ beweis der häufig herangezogen wird dass die silbe eben doch (0.2) kleinste baustein

Studentin: genau s is auch $n$ beweis dafür dass die silbe doch irgendwie im (.) mentalen lexikon mit abgespeichert sein muss

Prüferin/Studentin: ja/irgendwie

Studentin: (.) und dass es ne (.) also doch ne gewisse rolle spielt dabei

Prüferin: $\boldsymbol{h m}$ - $h \boldsymbol{m}$

\subsection{Aufmerksamkeitssignale: ja und $\mathrm{hm} h \mathrm{hm}$}

Vergleicht man die akustischen Parameter der Zustimmung mit den anderen Realisierungen von ja und $h m \_h m$, wird deutlich, dass sie zu unterschiedlichen kommunikativen Zwecken verwendet werden. Im Verhältnis zum Zustimmungssignal charakterisiert sich $j a$ in der Funktion eines aufmerksamkeitsbezeugenden Signals durch eine steigende Grundfrequenz (von ca. 177 bis $231 \mathrm{~Hz}$ ), eine fallende Intensität (von ca. 73 bis $59 \mathrm{~dB}$ ) sowie eine längere Dauer (ca. 0,3 Sek.), vgl. Graphik 3. 


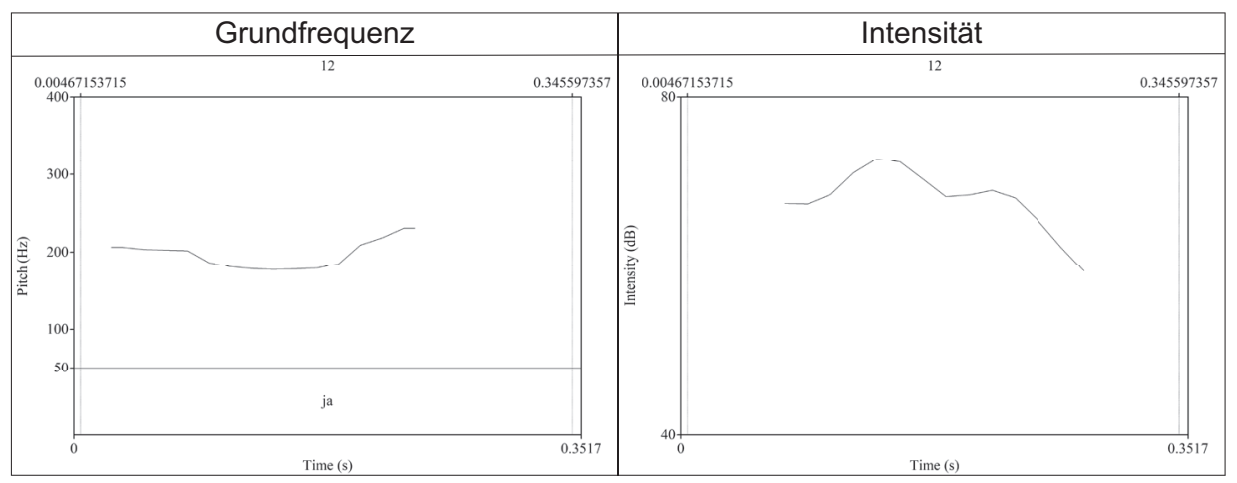

Graphik 3: Grundfrequenz- und Intensitätskonturen von ja in der Funktion eines Aufmerksamkeitssignals

Die Prüferin bekundet Interesse am Redebeitrag des Prüflings, was insbesondere durch die steigende Intonation ${ }^{13}$ und die vokalische Dehnung markiert wird. Das Aufmerksamkeitssignal erfolgt direkt nach einer als steigend wahrgenommenen Intonationsphrase vonseiten der Studentin. In dieser Form verliert $j a$ seine lexikalische Bedeutung und tendiert zum Ausdruck der Reziprozität, vgl. Dialog 3.

Dialog 3

Studentin: un es is n modulares und (0.3) serielles (.) modell (0.7) im gegensatz zu dem andern (.) ähm von (.) (susan caroll),

Prüferin: ja

Studentin: das is eher ein (.)

Prüferin: ((räuspert sich))

Studentin: (.) also das ist ein modular (0.4) interaktives modell (.) wo einfach der unterschied is also bei dem interaktiven dass bottomup und

Prüferin: $h m \_h m$

Studentin: topdownprozesse gleichzeitig laufen können

In derselben Funktion kommt $h m \_h m$ vor: Die Grundfrequenz steigt (von ca. 216 bis $346 \mathrm{~Hz}$ ), die Intensität sinkt (von ca. 70 bis $61 \mathrm{~dB}$ ) und die Dauer liegt bei ca. 0,3 Sek. In den beiden Fällen erscheinen die Aufmerksamkeitssignale als unmittelbare Reaktion auf den hohen Grenzton am Ende der studentischen Intonationsphrase, vgl. Graphik 4 und Dialog 4.

13 Im Transkript zusätzlich durch Komma markiert. 


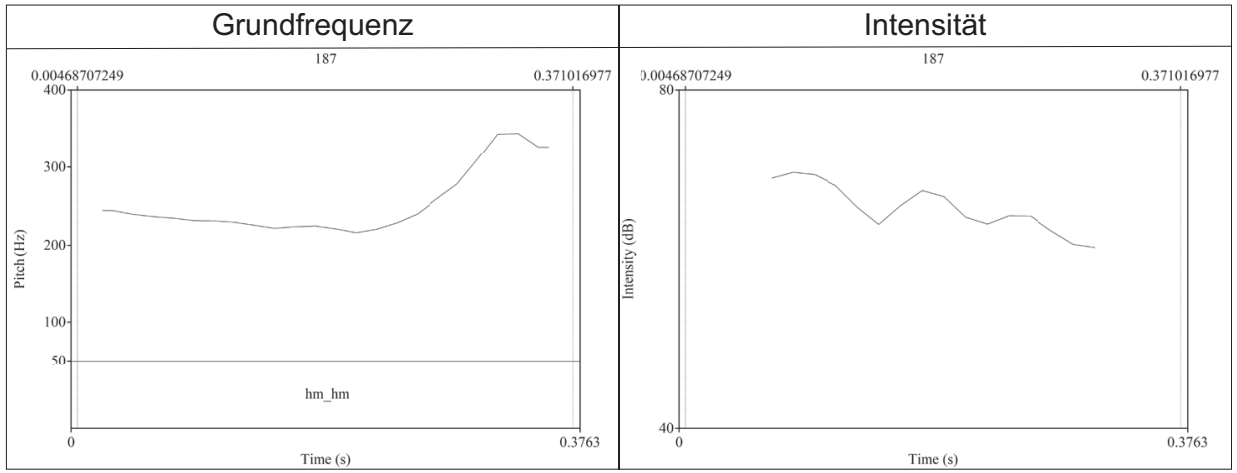

Graphik 4: Grundfrequenz- und Intensitätskonturen von $h m_{-} h m$ in der Funktion eines Aufmerksamkeitssignals

Dialog 4

Studentin: und dann waren die lerner dranne und ham die sachen natürlich (.) doch nochmal falsch ausgesprochen wie_s oft so is

Prüferin: $j a$

Studentin: ${ }^{\circ} \mathrm{h}$ die lehrerin hat sie dann korrigiert und die lerner waren dann also die ham das selbst mitgekricht zum einen schon mal

Prüferin/Studentin: $h m \_h m / a l s o$

Studentin: (.) des äh dass da $n$ fehler mit dabei war,

Prüferin: (.) hm_hm

Studentin: ham das dann auch mehrmals versucht (.) auszusprechen nach $m$ dritten mal war_s dann auch in etwa richtig aber nach zehn minuten war die (.) also war das quasi wieder schon hin

Die Aufmerksamkeitssignale machen ca. 14\% der untersuchten Rezeptionssignale aus. Dabei werden sie vorwiegend mit ja produziert, das etwas länger und leiser als $j a$ in der Funktion eines Zustimmungssignals ausgesprochen wird.

\subsection{Ermunterungssignale: ja und $\mathrm{hm} h \mathrm{hm}$}

Die nächste Realisierung von $j a$ zeichnet sich durch eine hohe Dynamik der Grundfrequenz (Anstieg von ca. 167 bis $352 \mathrm{~Hz}$ ) und der Intensität (Abstieg von ca. 69 bis $42 \mathrm{~dB}$ ) aus. Die Dauer liegt über 0,4 Sek., vgl. Graphik 5. 


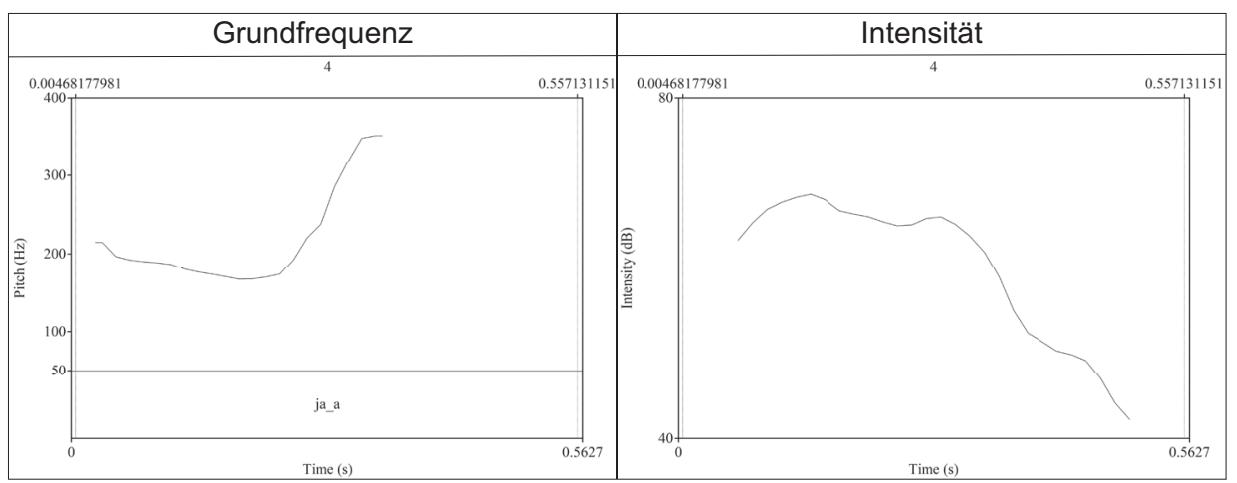

Graphik 5: Grundfrequenz- und Intensitätskonturen von $j a$ in der Funktion eines Ermunterungssignals

Ähnliches wird bei $h m_{-} h m$ festgestellt: eine hochsteigende Grundfrequenz (von ca. 181 bis $285 \mathrm{~Hz}$ ), eine tief fallende Intensität (von 64 bis $43 \mathrm{~dB}$ ) und eine längere Dauer (von ca. 0,4 Sek.), vgl. Graphik 6.

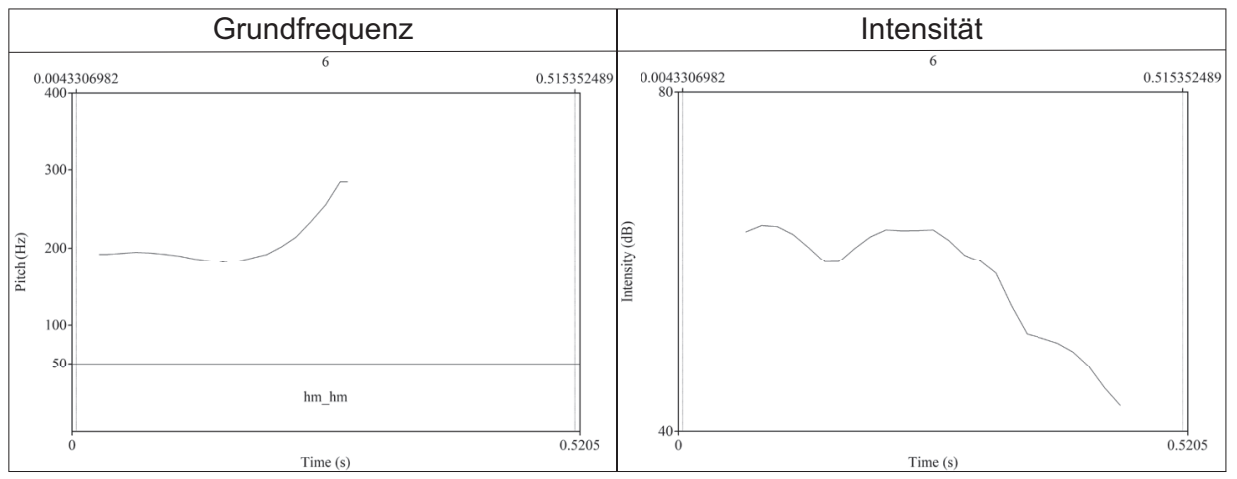

Graphik 6: Grundfrequenz- und Intensitätskonturen von $h m \_h m$ in der Funktion eines Ermunterungssignals

Durch die prosodischen Mittel (der hohe Tonhöhensprung, die immer niedrigere Lautstärke, die längere Dauer) drückt die Prüferin nicht nur die bloße Neugier aus - wie im Falle der Aufmerksamkeitssignale - sondern ermuntert die Studentin weiterzusprechen. Eine entscheidende Rolle spielt dabei die hoch steigende Tonhöhe, die anzeigt, dass eine Fortführung erwartet wird. Peters (2014:58) weist nach, dass der hohe finale Grenzton „konversationelle Unabgeschlossenheit“, während der tiefe finale Grenzton die ,potenzielle Abgeschlossenheit“ signalisieren kann. Solch eine gesprächsorganisatorische Strategie wird von beiden Kommunikationsakteuren angewendet. Die Prüferin benutzt die derart markierte Intonation, wenn sie die Studentin anregen will, die Aussage zu erweitern. Die Kombination der beiden Signale befindet sich im Dialog 5 (vgl. unten). In der 
GAT 2-Transkription wird ja in der Funktion des Ermunterungssignals mit ja $a$ wiedergegeben. Es wird nämlich zwischen $j a$ und $h m$ als „einsilbigen“ und ja_a und $h m \_h m$ als ,zweisilbigen“ Rezeptionssignalen unterschieden. Allerdings zeigen die akustischen Daten, dass das analysierte $J a$ aus einer Silbe besteht, deren Gipfel eine heterogene phonetische Struktur aufweist.

Dialog 5

Prüferin: also (0.2) wenn sie die so vergleichen (.) also jetzt nich lückenlos das system (.) sondern (0.2) was äh (.) bieten die beiden (0.3) modelle (0.3) für (0.3) äh (.) für das thema artikulation oder aussprache

Studentin: ((schmatzt)) okay (.) also zum ersten das (0.3) leveltmodell oder levelt,

Prüferin: ja_a

Studentin: wie auch immer es genannt wird ${ }^{\circ} \mathrm{hh}$ ähm (.) also das modell wird ja sehr oft

Prüferin/Studentin: ja_a/rezipiert

Studentin: einfach auch in allen psycholinguistischen einführungen (.) bei allen möglichen studien

Prüferin: $\boldsymbol{h m}$ h $h \boldsymbol{m}$

Studentin: und ähm ${ }^{\circ} \mathrm{h}$ also dieses modell bietet einfach (.) also (s) is (sehr) detailliert (.) auch im bezug auf die (.) artikulation (.)

Ca. 11\% der untersuchten Rezeptionssignale werden als Ermunterungssignale eingestuft. Es wird dabei hauptsächlich $j a$ eingesetzt. Die Ermunterungssignale sind deutlich länger und leiser als die Aufmerksamkeitssignale.

\subsection{Bestätigungssignale: $h m$ und ja}

Bei der letzten Gruppe handelt es sich um die Signale $h m$ und ja, die bestätigen, dass die Prüferin dem Redebeitrag der Studentin hinhörend folgt. Im Verhältnis zu den oben genannten Rückmeldungen haben die Bestätigungssignale eine recht neutrale phonetische Form, d. h. eine gleichbleibende Grundfrequenz, eine gleichbleibende Intensität und eine kurze Dauer. Die Grundfrequenz von $\mathrm{hm}$ liegt zwischen ca. 185 und $208 \mathrm{~Hz}$, die Intensität zwischen 61 und $63 \mathrm{~dB}$ und die Dauer bei ca. 0,2 Sek. Die Bestätigungssignale werden zumeist in die Aussage der Studentin eingeschlossen, vgl. Graphik 7 und Dialog 6. 


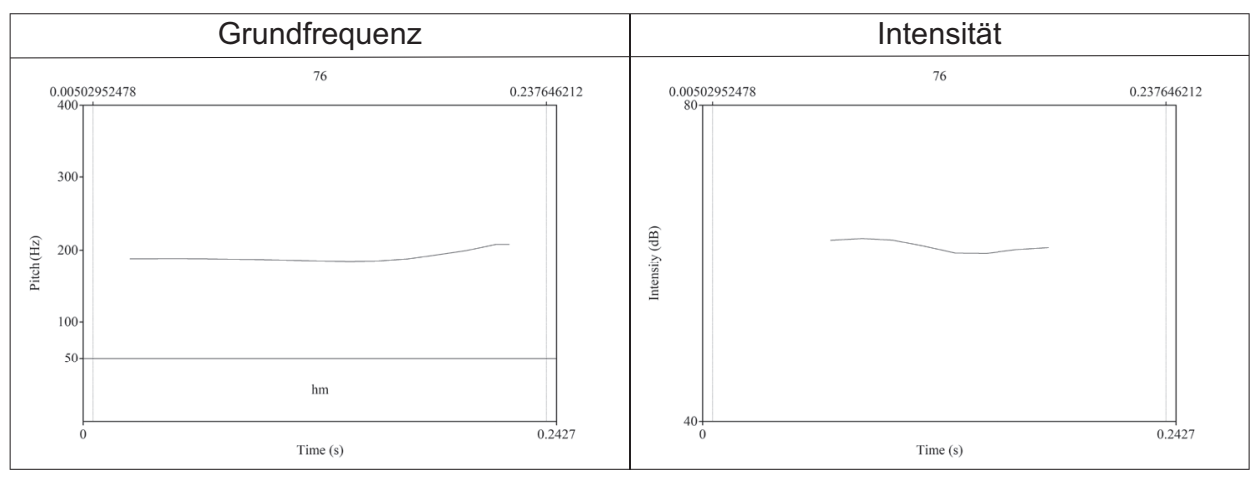

Graphik 7: Grundfrequenz- und Intensitätskonturen von $h m$ in der Funktion eines Bestätigungssignals

Dialog 6

Studentin: ich glaube das hängt schon doch mitnander zusammen weil d (.) eine annahme ist nämich ${ }^{\circ} \mathrm{h}$ dass äh die metrische struktur aus der segmentalen (.) zu ziehen is (.) weil (.) also s es so $n$ grundbetonungsmuster gibt Prüferin: (.) $\mathbf{h m}$

Studentin: in (.) jeder sprache also zum beispiel silbenzählend oder (0.3) taktzählend

Die akustischen Werte von $j a$ sehen ähnlich aus: die Grundfrequenz von ca. 191 bis $201 \mathrm{~Hz}$, die Intensität von ca. 60 bis $66 \mathrm{~dB}$, eine kurze Dauer von ca. 0,2 Sek. Die Bestätigungssignale werden viel leiser als die Zustimmungs- oder Aufmerksamkeitssignale realisiert. Sie treten beiläufig innerhalb des studentischen Redebeitrags auf, ohne dabei einen negativen Einfluss auf seine Struktur zu haben. Dadurch wird der Kontakt zur Sprecherin bestätigt und das aktive Zuhören demonstriert. Darüber hinaus ratifiziert die Art von Rezeptionssignalen das Recht der Studentin weiterzusprechen, vgl. Graphik 8 und Dialog 7.

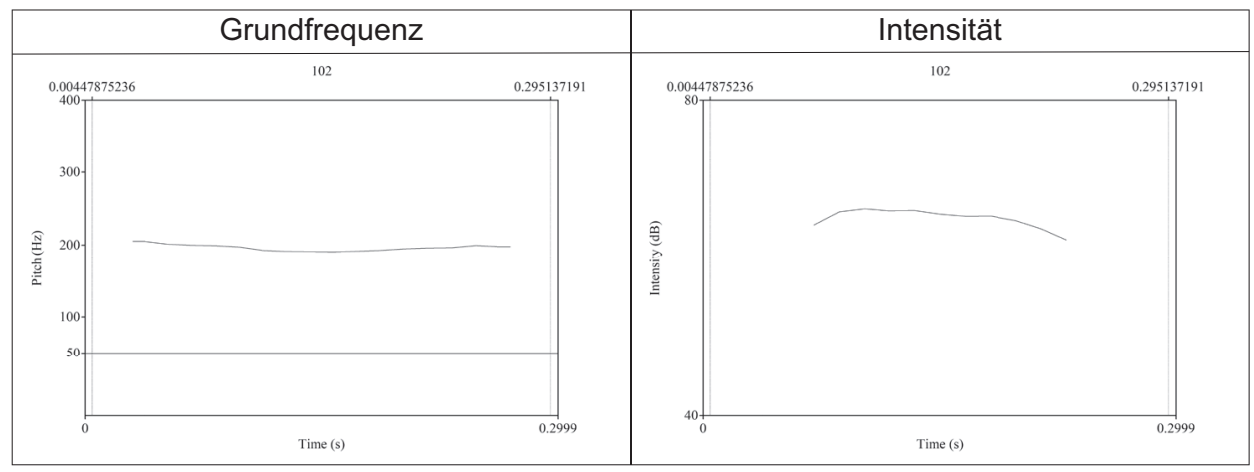

Graphik 8: Grundfrequenz- und Intensitätskonturen von ja in der Funktion eines Bestätigungssignals 


\section{Dialog 7}

Studentin: also ich fand halt besonders intressant an der sache und deswegen hab ich das mit aufgenommen das wurde im seminar von (.) einer (.) ähm (0.7) also (.) einer (.) kollegin von mir mitgesagt,

Prüferin: ja

Studentin: ${ }^{\circ} h$ (.) die meinte dass äh viele leute wenn die im sprachlabor sind (.) $\ddot{a} \mathrm{hm}$ dass die dann ${ }^{\circ} \mathrm{h}$ (wenn die immer) dieses (.) also die (0.7) alles lesen und dann versuchen nachzusprechen (0.2) dabei große probleme ham und immer wieder die gleichen (0.2) fehler im prinzip machen oder immer wieder die gleiche aussprache doch falsch machen

Die Bestätigungssignale sind mit 23\% die zweit häufigste Gruppe der Rezeptionssignale im analysierten Prüfungsgespräch. Zwei Drittel davon werden mittels $\mathrm{hm}$ mitgeteilt.

\section{Zusammenfassung und Schlussfolgerungen}

In der sprechwissenschaftlichen Untersuchung zu den Rezeptionssignalen im Prüfungsgespräch konnte gezeigt werden, dass unterschiedliche semantische Nuancen von ja, hm_ hm und $h m$ aus dem pragmatischen und prosodischen Kontext deutlich wurden. Die phonetische Analyse hat Folgendes ergeben:

1. Je nach phonetischer Form werden unterschiedliche Bedeutungen denselben sprachlichen Einheiten zugeschrieben: Ja kann als Zustimmungs-, Aufmerksamkeits-, Ermutigungs- oder Bestätigungssignal und $h m_{-} h m$ als Zustimmungs-, Aufmerksamkeits-, oder Ermutigungssignal verwendet werden. Ausschlaggebend ist dabei die Tonhöhe, die den höchsten prosodischen Rang hat. Nichtsdestoweniger werden markante Unterschiede in der Länge und in der Lautstärke erkannt, die die interaktiven Funktionen der einzelnen Realisierungen mitbestimmen.

2. Je nach kommunikativer Absicht weisen unterschiedliche Rezeptionssignale phonetische Gemeinsamkeiten auf: Den Zustimmungssignalen $j a$ und $h m \_h m$ ist eigen, dass sie eine leicht fallende Grundfrequenz, eine tief fallende Intensität und eine relativ kurze Dauer aufweisen. Präferiert wird die implizite Form von $h m \_h m$ (mit dem Verhältnis 40:28). Demzufolge ist $h m \_h m$ in der Funktion der Zustimmung das häufigste Rezeptionssignal im gesamten Prüfungsgespräch. Eine vergleichbare prosodische Struktur weisen zudem andere lexikalische Einheiten auf, beispielsweise okay und genau.

3. Bei den Aufmerksamkeitssignalen ja und $h m_{-} h m$ steigt die Grundfrequenz und die Intensität sinkt. Das aufmerksamkeitsbezeugende $J a$ ist dabei etwas länger als das $J a$ in der Funktion des Zustimmungssignals. Die Aufmerksamkeitssignale drücken die Anteilnahme der Prüferin an den Inhalten aus, auf die sich die Studentin bezieht. Dadurch wird mitgeteilt, dass die Studentin ihr Redebeitrag 
ungestört fortsetzen kann. Das Interesse wird viel häufiger mittels ja als $\mathrm{hm}$ - $\mathrm{hm}$ zur Sprache gebracht (Verhältnis 15:4).

4. Im Vergleich mit den Aufmerksamkeitssignalen zeigen die Ermutigungssignale ja und $h m \_h m$ eine noch höher steigende Grundfrequenz, eine tief fallende Intensität und eine noch längere Dauer auf. Dabei werden sie leiser als die $\mathrm{Zu}-$ stimmungs- und die Aufmerksamkeitssignale produziert. Die Ermutigungssignale ähneln sowohl akustisch als auch auditiv einer Frage, was bewirkt, dass die studentische Aussage anschließend erweitert wird. Der Hinweis weiterzusprechen wird fast ausschließlich durch $j a$ ausgedrückt (Verhältnis 13:2).

5. Im Gegensatz zu den Zustimmungs-, Aufmerksamkeits- und Ermunterungssignalen nehmen die Bestätigungssignale eine recht neutrale phonetische Form an. Sowohl die Grundfrequenz als auch die Intensität sind relativ konstant. Dabei sind die Bestätigungssignale kurz und haben eine niedrige Lautstärke. Sie sind ein wichtiges Signal für die Studentin, dass sie ihr Rederecht behalten kann oder soll. In dieser Funktion werden $h m$ und $j a$ (mit dem Verhältnis 20:10) verwendet.

\section{Literatur}

Bartsch Elmar / PABSt-Weinschenk Marita, 2011, Gesprächsführung, in: Pabst-Weinschenk M. (Hrsg.), Grundlagen der Sprechwissenschaft und Sprecherziehung, München, S. 123-132.

BRINKER Klaus / CöLFEN Hermann / PAPPERT Steffen, 2014, Linguistische Textanalyse. Eine Einführung in Grundbegriffe und Methoden, Berlin.

Brock Alexander / MeER Dorothee, 2004, Macht - Hierarchie - Dominanz - A-/Symmetrie: Begriffliche Überlegungen zur kommunikativen Ungleichheit in institutionellen Gesprächen, in: Gesprächsforschung - Online-Zeitschrift zur verbalen Interaktion, Ausgabe 5, S. 184-209.

Bussmann Hadumod (Hrsg.), 2008, Lexikon der Sprachwissenschaft, Tübingen.

Cirko Lesław, 2016, Phatische Signale in deutschen, englischen und polnischen Prüfungsgesprächen, in: Sprachreport Jg. 32, Heft 1, S. 26-34.

Dudenredaktion (Hrsg.), 2009, Duden. Die Grammatik. Unentbehrlich für richtiges Deutsch, Band 4, Mannheim/Wien/Zürich.

EHLICH Konrad, 1986, Interjektionen, in: Linguistische Arbeiten, 111, Tübingen.

Gilles Peter, 2005, Regionale Prosodie im Deutschen. Variabilität in der Intonation von Abschluss und Weiterweisung, Serie: Linguistik - Impulse \& Tendenzen, Berlin.

GLÜCK Helmut / RöDEL Michael (Hrsg.), 2016, Metzler Lexikon Sprache, Stuttgart.

Graefen Gabriele / LiedKe Martina, 2008, Germanische Sprachwissenschaft. Deutsch als Erst-, Zweit- oder Fremdsprache, Tübingen.

Grassegger Hans, 2010, Phonetik / Phonologie, Idstein.

GüNTHER Carsten, 1999, Prosodie und Sprachproduktion, Tübingen.

HirschFeld Ursula / Stock Eberhard, 2013, Sprechwissenschaftliche Phonetik, in: Bose I./Hirschfeld U./Neuber B. (Hrsg.), Einführung in die Sprechwissenschaft. Phonetik, Rhetorik, Sprechkurs, Tübingen, S. 27-80.

Imo Wolfgang / LANwer Jens Philipp, 2019 Interaktionale Linguistik. Eine Einführung, Stuttgart. JANICH Nina / BirKner Karin, 2015, Text und Gespräch, in: Felder E./Gardt A. (Hrsg.), Handbuch Sprache und Wissen, Berlin/Boston, S. 195-220. 
Linke Angelika / Nussbaumer Markus / Portmann Paul R., 2004, Studienbuch Linguistik, Tübingen.

MeER Dorothee, 1998, Der Prüfer ist nicht der König. Mündliche Abschlußprüfungen in der Hochschule, Tübingen.

Neuber Baldur, 2013, Gespräch, in: Bose I./Hirschfeld U./Neuber B./Stock E. (Hrsg.), Einführung in die Sprechwissenschaft. Phonetik, Rhetorik, Sprechkunst, Tübingen, S. 114-120.

Peters Jörg, 2014, Intonation, Heidelberg.

PÉTURsson Magnús / NepPerT Joachim M.H., 2002, Elementarbuch der Phonetik, Hamburg.

RATH Reiner, 1979, Kommunikationspraxis. Analysen zur Textbildung und Textgliederung im gesprochenen Deutsch, Göttingen.

Schegloff Emanuel A., 1982, Discourse as an interactional achievement: Some uses of , uh huh and other things that come between sentences, in: Tannen D. (Hrsg.), Analyzing Discourse: Text and Talk, Washington, S. 71-93.

Schwitalla Johannes, 2012, Gesprochenes Deutsch. Eine Einführung, Berlin.

Selting Margret, 1995, Prosodie im Gespräch. Aspekte einer interaktionalen Phonologie der Konversation, Tübingen.

SeLTING Margret, 2001, Berlinische Intonationskonturen: ,Die Treppe aufwärts‘, in: Zeitschrift für Sprachwissenschaft, 20 (1), S. 66-116.

SteIN Stephan, 2003, Textgliederung. Einheitenbildung im geschriebenen und gesprochenen Deutsch: Theorie und Empirie, Berlin/New York.

Sucharowski Wolfgang, 2001, Gespräche in Schule, Hochschule und Ausbildung, in: Brinker K./ Antos G./Heinemann W./Sager S.F. (Hrsg.), Text- und Gesprächslinguistik. Ein internationales Handbuch zeitgenössischer Forschung, 2. Halbband, Berlin/New York, S. 1566-1576.

TeсhтmeIER Bärbel, 1998, Fachtextsorten der Wissenschaftssprachen VII: das Prüfungsgespräch, in: Hoffmann L./Kalverkämper H./Wiegand H.E. (Hrsg.), Fachsprachen. Ein internationales Handbuch zur Fachsprachenforschung und Terminologiewissenschaft, 1. Halbband, Berlin/ New York, S. 517-521.

TitTtula Liisa, 2001, Formen der Gesprächssteuerung, in: Brinker K. /Antos G./Heinemann W./Sager S.F. (Hrsg.), Text- und Gesprächslinguistik. Ein internationales Handbuch zeitgenössischer Forschung, 2. Halbband, Berlin/New York, S. 1361-1374.

\title{
Internetseitenverzeichnis
}

https://exmaralda.org/de/.

https://gewiss.uni-leipzig.de/.

http://www.fon.hum.uva.nl/praat/.

http://www.gespraechsforschung-ozs.de/heft2009/px-gat2.pdf.

\section{Anhang}

\author{
tionen \\ (.) \\ (0.4) \\ - Mikropause, geschätzt, bis ca. 0,2 Sek. Dauer \\ - gemessene Pause von ca. 0,4 Sek. Dauer \\ - steigende Tonhöhenbewegung
}

Verwendete Transkriptionszeichen nach den GAT2-Transkriptionskonven- 
; $\quad-$ fallende Tonhöhenbewegung

${ }^{\circ} h \quad-$ hörbares Einatmen von ca. 0,2-0,5 Sek. Dauer

$(($ schmatzt $)) \quad-$ nonverbale Handlungen und Ereignisse

\section{Reception signals in an exam interview from the spoken communication point of view}

The article aims to describe the reception signals present in the German exam interview from the spoken communication point of view. Short (one- and two-syllable) feedback signals will be analysed, which represent the examiner's attitude towards the statement heard. More specifically, the forms are "ja", "hm_hm" and "hm", which, depending on the prosodic implementation, may have different interactive functions. The analysis will take into account acoustic-auditive parameters: fundamental frequency/pitch, intensity/volume and duration/length. On this basis, four types of reception signals have been identified: confirmatory signals, signals of interest, signals encouraging to continue speaking, and perceptual signals. It has been shown that, depending on their phonetic realisation, the same signal can perform several different communication functions, and that individual signals have a similar phonetic form depending on their function during the exam interview.

Keywords: reception signals, exam interview, prosody, acoustic-auditive parameters: fundamental frequency/pitch, intensity/volume, duration/length. 